\title{
Correspondence
}

TO THE EDITOR, British Journal of Venereal Diseases

\section{Conjunctivitis due to Haemophilus ducreyi infection}

\section{Sir,}

Haemophilus ducreyi is an extremely rare cause of ocular infections. ${ }^{1}$ This organism, which was described by Ducrey in 1889 , is the aetiological agent of soft chancre or chancroid-a venereal disease. The natural reservoir of this small pleomorphic Gramnegative bacillus is the genitourinary tract of man. In civilian populations this bacterium may be responsible for up to $10 \%$ of reported cases of chancroid. 2

A 25-year-old male medical student complained of acute conjunctivitis within four days of performing vaginal examinations on five female patients. His chief complaint was a pulsating ache and erythema of the conjunctiva of the left eye. There was also puffiness and tenderness of the upper lid of this eye and pain on attempted use of the extraocular muscles of the eye. A profuse yellow mucoid exudate was present in the medial punctum of the eye.

On closer examination it was noted that the scleral blood vessels were dilated with haemorrhage from some of these vessels. An enlarged non-tender left preauricular lymph node and a slightly enlarged nontender submaxillary gland were detected by palpation. A presumptive diagnosis of epidemic keratoconjunctivitis, probably due to adenovirus, was made based on the clinical picture presented.

Approximately one hour after examination by an ophthalmologist and before treatment with an ophthalmic solution (naphazoline $\mathrm{HCl}$ in a dosage of 2-3 drops) into the infected eye in conjunction with cold compresses to reduce swelling a portion of the exudate was collected for culture using a cotton swab. A sheep-blood agar plate and a chocolate agar plate were inoculated and both incubated in a candle jar for 24 hours at $37^{\circ} \mathrm{C}$. After this period a few identical tiny greyish translucent colonies were observed on both media; however, more colonies appeared on the chocolate agar than on the blood agar plate. A Gram stain of a few colonies from both media showed pleomorphic Gramnegative bacilli, morphologically consistent with the genus Haemophilus.

Three colonies from each medium were inoculated on to separate trypticase soy agar (TSA) plates; a V factor (nicotinamide adenine nucleotide) strip and an $X$ factor (hemin) strip (Baltimore Biological Laboratory, Cockeysville, Maryland, USA) were placed on both plates. The plates were incubated under $\mathrm{CO}_{2}$ tension in a candle jar at $37^{\circ} \mathrm{C}$ and examined after 24 hours.

Three colonies were also transferred to a test tube containing $10 \mathrm{ml}$ of nitrate broth (Difco Laboratories, Detroit, Michigan, USA) and incubated for 24 hours at $37^{\circ} \mathrm{C}$. The TSA plates showed tiny refractile colonies growing around the $\mathrm{X}$ factor strip; no growth occurred around the $\mathrm{V}$ factor strip. No change was observed in the nitrate broth indicating that the organism did not reduce nitrate to nitrite. The organism was identified as Haemophilus ducreyi.

After 11 days' treatment with the ophthalmic solution and cold compresses the patient became asymptomatic and has not experienced any recrudescence. Yours faithfully,

$J$ E Gregory $R W$ Henderson $R$ Smith

Departments of Microbiology and

Ophthalmology,

Howard University Hospital and

College of Medicine,

Washington DC,

USA

\section{References}

1. Fedukowicz HB. External Infections of the Eye. 2nd ed. New York: Appleton-CenturyCrofts, 1978: 160-1

2. Joklik WK, Willett HP. Zinsser Microbiology, 16th ed. New York: AppletonCentury-Crofts, 1976:471-8.
TO THE EDITOR, British Journal of Venere® Diseases

\section{Trichomonal urethritis in men}

Sir,

I would like to offer a comment on you $\overline{\mathrm{T}}$ recent articlel on the investigation of trichomonal urethritis in men. The technique was shown to be sensitive and capable of demonstrating small numbers of trichomonads in a urine specimen? However, when 18 of 21 male contacts of women known to have trichomonat vaginitis produced negative culture results the only conclusions offered were "that most male contacts shed relatively few trichomonads and that the infective dose for women must be correspondingle small."

May I suggest as an alternative, and more likely, conclusion that trichomonads werg not found in these 18 men because the were not present in the first place.

This report would appear to confirm $m$ suggestion $^{2}$ that trichomonal vaginitis is noD (necessarily) a sexually transmitted disease. Yours faithfully,

Genitomedical Department, $N$ Rosedale

Hillingdon Hospital,

Uxbridge, Middlesex

\section{References}

1. Wilson A, Ackers JP. Urine culture for the detection of Trichomonas vaginalis in men
Br J Vener Dis 1980;56:46-8.

2. Rosedale N. Trichomonal vaginitis is not $\$$ venereal disease. World Medicine 1977; 12: 33 .

TO THE EDITOR, British Journal of Venered Diseases

Treatment of lymphogranuloma venereuhi with rifampicin

Sir,

With reference to the letter on the "Treat ment of lymphogranuloma venereum wit rifampicin"' by Menke and co-workers, ${ }^{1} \Phi$ 